\title{
On problem of the rigorous diffraction quantitative description
}

\author{
S. Anokhov \\ International Center «Institute of Applied Optics» of NAS of Ukraine \\ 10-G vul. Kudryavska, 254053, Kyiv, Ukraine, phone: (38044) 212-21-58; fax: (38044) 212-48-12, e-mail: khizh@lomp.ip.kiev.ua
}

\begin{abstract}
New data showing an inaccuracy of Kirchhoff's description for the diffraction of the limited aperture light beams are presented. A series of the known experimental facts, which did not have an unequivocal interpretation within the framework of this theory, gains a simple explanation with passage to conceptions of the rigorous diffraction theory. The difficulties hindering the wide application of this theory for solving practical problems are considered as well.
\end{abstract}

Keywords: diffraction, boundary wave, light beem, aperture diaphragm.

Paper received 28.09.99; revised manuscript received 30.11.99; accepted for publication 17.12.99.

\section{Introduction}

The present article is initiated by the Wang's publication «On principles of diffraction» [1], in which the author, proceeding from the existing experimental facts and results of his own investigation, comes to a conclusion about the necessity to correct the currently formed conception of diffraction. His generalization concerning a set of known effects (illogical position of axial intensity maxima in the Fresnel zone, focal spot displacement at convergent wave diffraction, etc.), quantitative explanation of which required to ascribe half-wave phase shift to diffracting wave edges relatively to a remaining wave, was the ground for this. According to Wang, to correct the situation it is enough to improve the former wave principle by including, once for all the indicated phase shift for secondary waves touching with an obstacle.

In this connection it is pertinently to remind about a natural phase shift of a diffracted wave that figures in the rigorous theory of diffraction and is not connected with the action of Huygens-Fresnel principle. The following section of the article is devoted to discussion of this problem. Besides this, the extra experimental facts, which do not fit to the Kirchhoff diffraction theory, are presented in the article. The problems hampering wide practical distribution of the rigorous theory of the diffraction are considered as well.

\section{The phase shift in the diffracted wave}

As is known, the formation of the modern conception of diffraction occurred in rivalry conditions of two alternate approaches to this phenomenon bound, accordingly, to names of Young and Fresnel. The rigorous solution of a problem of plane wave diffraction on an ideally conductive half-plane obtained by Sommerfeld in 1896 [2,3] became the decisive argument for the benefit of Young. According to it, the resulting field can be represented by a superposition of two components (Fig.1), namely, geometrical component $A_{G}$ (the remaining part of the initial wave, that suffers discontinuity and propagating further by rules of geometrical optics) and diffracting or boundary wave $A_{B}$ - divergent wave, geometrical center of which coincides with the edge of a half-plane.

One of the most typical distinctive feature of the boundary wave is the presence of the half-wave sudden change in phase in it that coincides with the boundary of the geometrical shadow. Thereof, these halves of the wavefront situated on different sides from the indicated boundary are counter-phase (Fig. 1). A simple explanation of this circum-

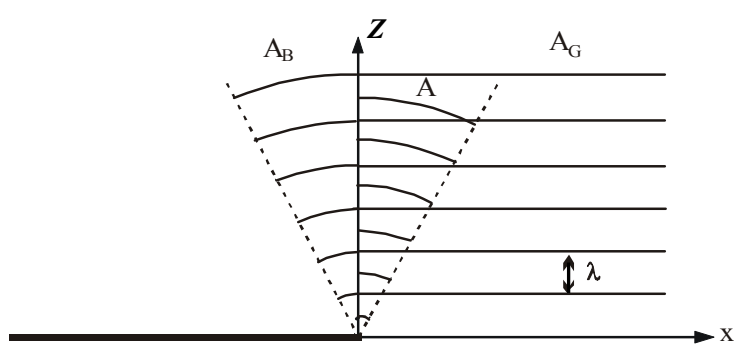

Fig. 1. Schematic explanation of diffraction field structure behind the screen based on rigorous diffraction theory. 


\section{S.Anokhov: On problem of the rigorous diffraction quantitative...}

stance made by Rubinovicz [4] consists in the following.

Sommerfeld solved the problem as a boundary one for the Maxwell equations. By virtue of this, the solution obtained by him, naturally, does not contain any discontinuities of the field on the boundary of a geometrical shadow. Otherwise, they should be connected to the presence of actual charges or currents here, for occurrence of which there are no physical grounds. As the incident wave undergoes the discontinuity on edge of the screen, the boundary wave should also have here the same effect, but of such kind, at which the discontinuity of the composite field disappears. The latter actually takes place due to the geometrical component, and the boundary wave has opposite phase when approaching to the boundary of a shadow from the illuminated side and the identical phase when approaching to it from the shadow one (Fig. 2).

The presence of such structure in the boundary wave completely corresponds to the results of experimental observations [3-9]. Thus, at the electromagnetic wave diffrac-
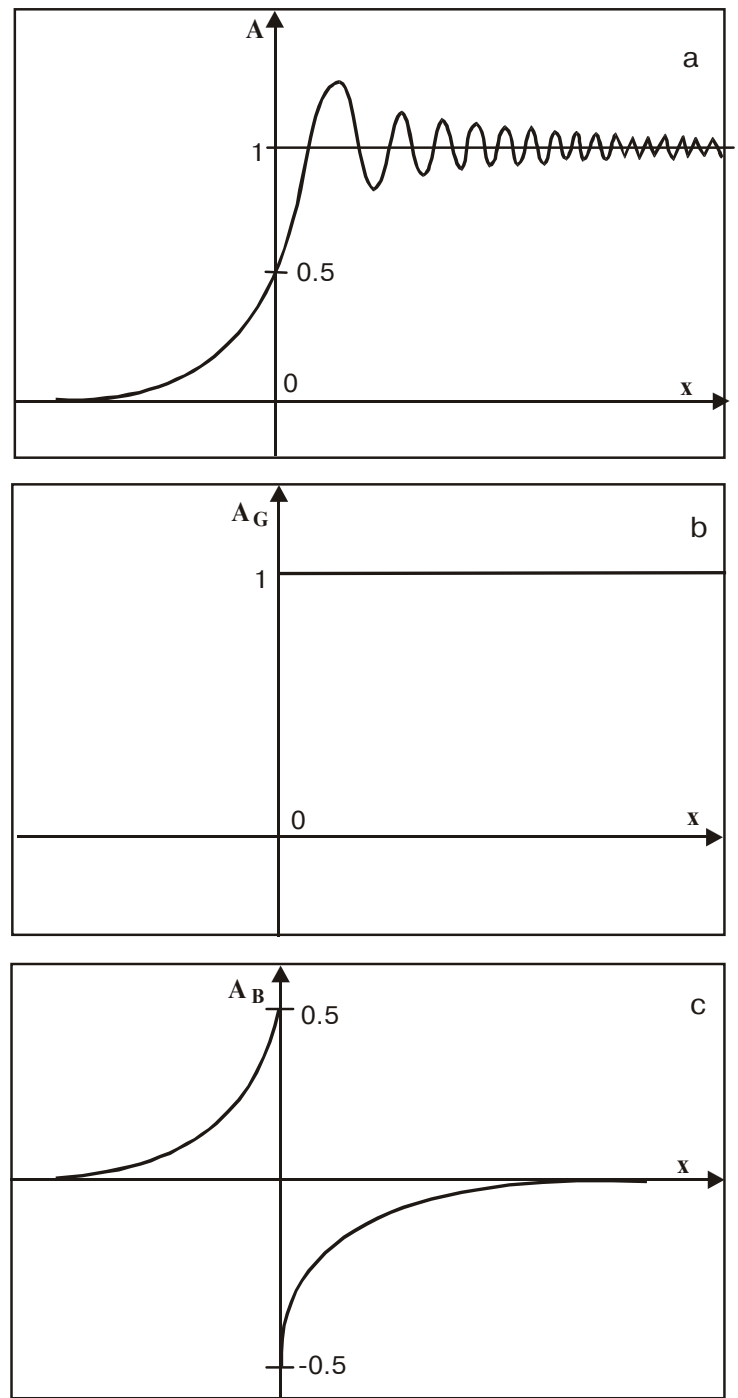

Fig. 2. Addition (a) of amplitudes of geometrical (b) and boundary (c) waves at edge diffraction of a plane wave. tion on an arbitrary screen in the illuminated part of space there always exists the divergent wave propagating from an edge of the screen and $\pi$ phase shifted relatively to the basic wave. The explanation of the effects described in the paper [1] becomes obvious with accounting it.

However, the discussed problem is not exhausted, as there is no general quantitative description of the described phenomenon so far. Effectivelly, it is talked about the absence of the rigorous theory of aperture diffraction that would be capable to replace Kirchhoff's theory, which is not so reliable in this situation. The improvement of the wave principle by including of half-wave phase shift for secondary waves touching the obstacle, proposed in [1], can hardly solve the problem.

It is enough only to pay attention to the fact that the energy of selected counter-phase components in this case are many orders of magnitude lower than the remaining wave field, which excludes a possibility of their noticeable effect on its common structure. Let's remind, that in due time Fresnel has met the similar problem, trying to calculate the simplified Young's model [10]. This circumstance, as known, has pushed him to search other approach towards the diffraction.

\section{Far field anomalies}

According to the Kirchhoff theory, during the diffraction of an arbitrary wave on an aperture, the distribution of the field in the Fraunhofer zone is described by the Fourier transforms of the initial field distribution on the aperture. This transforms, in particular, lies in the basis of the modern theory of aperture antennas $[11,12]$. However, as careful measurements show, the indicated correspondence is fulfilled precisely only for the plane wave diffraction. With the deviation of the amplitude distribution of a plane (quasi-plane) wave at the aperture from homogeneous one, the resulting Fourier-transform more and more differs from the real far field pattern [13].

To estimate the scale of this discrepancy, let us turn to the results of the experiment on single-mode laser beam diffraction on a slit aperture, beam-waist of which was superposed with the plane of the slit when using a telescopic system. The latter enables to consider the wave front of the diffracting wave plane. Thus, we deal with the aperture diffraction of a non-uniform plane wave with the Gaussian profile of amplitude. The typical pattern of the far field intensity distribution observed in this case is shown in Fig. 3. For definiteness we shall consider such particular characteristic of this field as angular distribution of its minima. If one accepts as the initial reference point, the equidistant sequence of diffraction minima formed by the plane wave, and calculates the individual shifts of these minima after the replacement of the plane wave by an arbitrary non-uniform wave with the plane front, the common regularity arises [13]: the absolute shift of minima always decreases with moving from the center of the distribution (Fig. 4).

This rule, however, completely refutes the above mentioned experiment, demonstrating the opposite behavior of actual minima of the far field, of real shift which practically 


\section{S.Anokhov: On problem of the rigorous diffraction quantitative...}

always increases to the periphery of the distribution. The objective character of such field evolution was also confirmed by the author's analysis of accumulated in literature facts that concerns the diffraction of non-uniform waves, for several recent decades. Such deep discrepancy between the expected and actual structures of the far field specifies the presence of unknown physical factor at the diffraction process, which formerly escaped from the attention of the investigators.

To clarify its character, the computer simulation of functions, Fourier-transforms' shape of which would coincide with the observed pattern of the far field, was undertaken. As it was eventually found, the required field distribution at the slit can be composed as the sum of two components (Fig. 5), namely the diffracting wave itself and $\pi$ phase shifted wave, the most part of the power of which is concentrated in a pair of separate peaks, which are clasped to the edges of a slit [13]. For the shape and height of the peaks shown in Fig. 5, the highest resemblance between the calculated and observed structures of the final distribution, is practically reached for all quantitative indexes.

At the same time, the actual existence of a similar wave was not experimentally confirmed: in the field structure recorded using CCD-camera just behind the slit, any differences from initial distribution of the diffracting wave were not found. Thus, the introduction of a new wave in this case should be consider, only as formal technique for the solution of the particular problem which makes it possible to remove the arisen quantitative contradiction. The final clearness into the situation can be brought only by the sequential solution of this problem within the framework of the rigorous theory of diffraction, which persist on half-wave phase shift for a part of the wave field, as the logic link of the diffraction process.

\section{Fundamental problems of the rigorous theory}

The preference that is given to the Kirchhoff's theory, when the practical case is under the consideration, can be explained not only through its clearness and simplicity. Although being rehabilitated by Sommerfeld, the Young's concept corresponds to currently most rigorous quantitative approach to the diffraction, and it has lead also to some unexpected problems. In particular, this concept is in a contradiction with the so-called condition on a rim («a rim does not radiate»), the idea that has been accepted for the rigorous solution of the electrodynamics boundary-value problems [14]. As a result, this concept has not yet found a complete understanding. No less serious questions are caused by the analytical representation of the diffraction field that is apparent from this conception.

The given representation of the Sommerfeld solution in the form of two components did not receive the complete physical interpretation. In the area behind the screen both geometrical and boundary waves possess an amplitude break on the boundary of geometrical shadow. Moreover, the boundary wave has broken derivative of the transversal amplitude distribution and non-smooth wavefront. Being intuitively understandable, these waves can not exist and

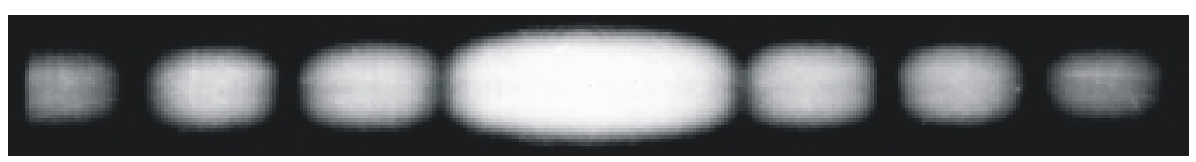

Fig. 3. Distribution of a real far field at aperture (slit) diffraction of gaussian beam.

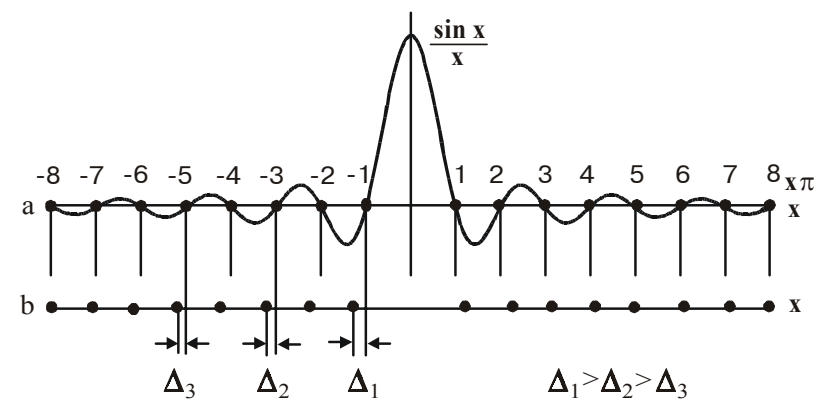

Fig. 4. The far field minima distribution for diffraction of an uniform plane wave (a) and an arbitrary light beam (b).

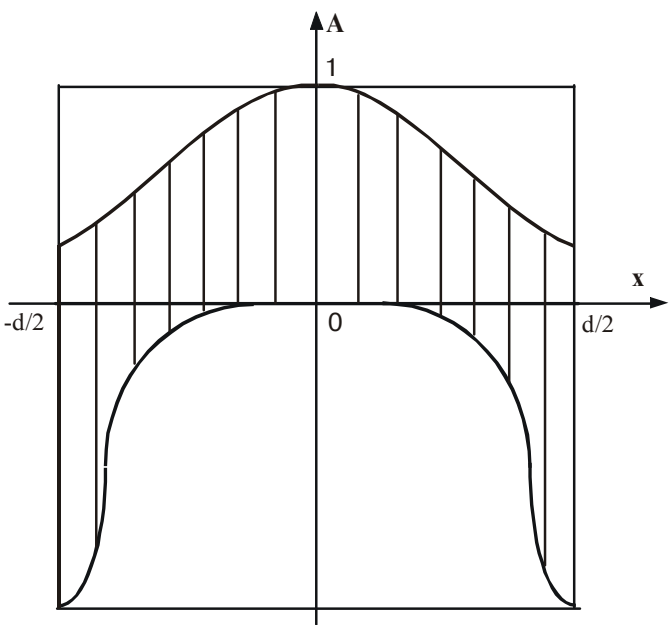

Fig. 5. The amplitude profile of the combined wave on the slit established by computer simulation ( $d$ designates the width of the slit). 


\section{S.Anokhov: On problem of the rigorous diffraction quantitative...}

propagate as real waves. Separately they do not satisfy wave equation, and the only their combination gives a proper solution. However, there exists a set of reports [3-9] describing an isolation and study of the boundary wave itself, but even up to now there is not any reasonable explanation of the physical reality of geometrical and boundary waves, thus putting questions about the validity of the solution.

Essentially, all the above listed moments represent almost insurmountable psychological barrier that severs a rigorous diffraction theory from the practical optics, the latter always being bearing up against the physically clear conceptions and laconic mathematical means. It is reasonable to notice that all given above and difficult for interpretation facts usually are omitted in literature on diffraction. To the best knowledge of the author, there are few publications where this problem has been mentioned and its principle importance was acknowledged $[8,15]$. However, the further serious advancement in this domain without its fundamental solution is hardly possible.

\section{Conclusion}

As it is clear, there are several enough serious aspects of the diffraction, explanation of which within the framework of Kirchhoff's theory remains difficult. Besides, the phenomena mentioned in the paper [1] and observed in the case of the Fresnel diffraction, are necessary now to be included to the same series of the structural peculiarities when considering the field formed in the Fraunhofer zone at the aperture diffraction of a nonuniform quasi-plane wave. The differences between the observed field pattern and the structure calculated by the traditional way are so significant that even require the improvements of the model lying in the basement of the conventional theory.

In particular, in this case it is not sufficient to have the usual expansions of the initial wave at the slit aperture onto plane waves ( Fourier transforms): for receiving the exact result it is necessary to supplement this wave by one more $\pi$ phase shifted, field maximums of which have to correspond to minima of the diffracting wave (Fig. 5). The Fourier-transform of such wave construction does represent the empirically found pre-image of rigorous solution of this problem that is absent up to date.

The author is very grateful to Prof. A. Khizhiyak for fruitful discussions and for his constructive remarks.

\section{References}

1. S. Wang, On principles of diffraction// Optik, 100, 107-108 (1995).

2. A. Sommerfeld, Mathematische Theorie der Diffraction// Math. Ann. 47, 317-374 (1896)

3. M. Born, E. Volf, Principles of optics // Pergamon Press, N.Y., (1970)

4. A. Rubinowicz, Thomas Young and the theory of diffraction// Nature 180, 162-164 (1957).

5. R.W. Wood, Physical optics // MacMillan Company, N.Y. (1934)

6. S. Ganchi, An experiment on the physical reality of edge-diffracted waves// Amer. J. Phys. 57, 370-373 (1989).

7. Yu. I. Terentiev, Diffraction of light on a thin flat screen with the straight edge // Optika Atmosf., 2, 1141-1146 (1989) (in Russian).

8. V. N. Smirnov, S. A. Strokovskii, On diffraction of optical Hermite-Gaussian beams from a diaphragm // Optika Spectrosk, 76, 1019-1026 (1994) (in Russian).

9. P. V. Polyanskii, G. V. Polyanskaya, On a consequence of the Young - Rubinowicz model of diffraction phenomena in holography» // Optica Applicata 25, 171-183 (1995).

10. O. Fresnel, Selected works in optics // GITTL, Moscow (1955), (in Russian).

11. R.C. Hansen, Aperture theory in Hansen R.C. (ed.) Microwave scanning antennas, v.1 Apertures // N.Y., Academic Press (1964)

12. J. W. Sherman, Aperture antennas theory in M.I. Skolnik (ed.) Radar handbook, 2, N.Y., Mc-Graw-Hill Book Company (1970).

13. S. Anokhov, Detailed study of a plane-plane cavity fundamental mode far field structure. // Proc. SPIE, 4018, p. 111-117 (1999)

14. H. Henl, A.W. Maue, K. Westpfahl, Handbuch der physik // Berlin, Springer - Verlag (1961).

15. R.B. Vaganov, B.Z. Kacenelenbaum, The basic of diffractional theory // Moscow, Nauka (1982), (in Russian). 